\title{
ASPECTOS CRÍTICOS CONTEXTUALES DE UN HOSPITAL DE ALTA COMPLEJIDAD
}

\author{
Adrián Torres Canales ${ }^{1}$, Álvaro Torres Gálvez², Miguel Bustamante Ubilla ${ }^{3}$, Sergio Contreras Villa
}

Resumen: Este artículo presenta los resultados parciales de un análisis FODA realizado en el Instituto Nacional del Tórax, con el fin de generar información sobre los aspectos críticos que afectan la gestión estratégica de un hospital público de alta complejidad, y abrir una reflexión ética sobre tales procesos. Para su determinación se usaron focus group, entrevistas en profundidad, propuestas por el equipo Directivo del Hospital y discusiones en torno al ámbito contextual. Las entrevistas individuales y grupales tuvieron una duración de una hora y veinte minutos aproximadamente y se realizaron en dependencias del hospital. La conceptualización de los datos, se hizo desde la Teoría Fundamentada. El estudio muestra, de acuerdo con la percepción de los entrevistados, que hay aspectos contextuales, tales como reglas del juego, stakeholders y cultura país, cuya tensión ética con la organización, afectan el quehacer del Instituto Nacional del Tórax, en cuanto hospital público de alta complejidad.

Palabras clave: análisis contextual, hospital público de alta complejidad, tensión ética de la función pública, impacto en la gestión, percepción de actores relevantes

\section{Critical contextual aspects of a highly complex hospital}

\begin{abstract}
This article presents the partial results of a SWOT analysis at the National Chest Institute, in order to generate information on critical issues affecting the strategic management of a public hospital of high complexity, and open an ethical reflection on such processes. For their determination were used: focus groups, interviews, proposed by the management team of the Hospital and discussions on the contextual level. The Individual and group interviews lasted about an hour and twenty minutes and were performed at hospital units. The conceptualization of the data was made from Grounded Theory. The study shows, according to the perception of the interviewees, their contextual issues such as rules, stakeholders and cultural country, whose ethical tension with the organization, affect the work of the National Chest Institute as a high complexity public hospital.
\end{abstract}

Key words: contextual analysis, high complexity public hospital, ethics tensions at public service, impact on management, perception of stakeholders

\section{Aspectos críticos contextuais de um hospital de alta complexidade}

Resumo: objetivo: Este artigo apresenta resultados parciais de uma análise FOFA realizada no Instituto Nacional de Tórax do Chile, com a finalidade de gerar informações sobre os aspectos críticos que afetam a gestão estratégica de um hospital público de alta complexidade, e gerar uma reflexão ética sobre tais processos. Método: para sua determinação fora utilizado o focus group, entrevistas em profundidade propostas pela equipe de gestão do hospital e discussóes acerca do escopo contextual. A conceitualização dos dados, foi feita a partir da Teoria Fundamentada. Conclusôes: o estudo demonstra que, de acordo com a percepção dos entrevistados, há aspectos contextuais, tais como as regras do jogo, stakeholders e a cultura do país, cuja tensão ética com a organização afetam o trabalho do Instituto Nacional do tórax, enquanto hospital público de alta complexidade.

Palavras-chave: análise contextual, hospital público de alta complexidade, a tensão ética do serviço público, impacto sob a gestão, percepção dos atores relevantes

\footnotetext{
${ }^{1}$ Facultad de Ciencias Médicas, Universidad de Santiago de Chile, Chile Correspondencia: adrian.torres@usach.cl

${ }^{2}$ Universidad Alberto Hurtado, Chile

${ }^{3}$ Facultad de Economía y Negocios, Universidad de Talca, Chile

${ }^{4}$ Facultad de Ciencias de la Salud, Universidad de Magallanes, Chile
} 


\section{Introducción}

La motivación principal de este trabajo es aportar elementos de análisis que permitan visibilizar los fenómenos relacionados con la gestión y las políticas públicas de salud en Chile post reforma del sector. En ese sentido se recogen elementos del fortalecimiento ético de las instituciones públicas en A.L.(1), de ética pública y transparencia(2), aspectos de la teoría de los stakeholders(3), del humanismo y la gestión en salud(4), de la percepción de la motivación en algunos hospitales de Chile(5), del diagnóstico realizado para el Plan de mejoraniento del Sector Salud realizado el año 2009(6); más recientemente, del trabajo realizado sobre la situación financiera de los hospitales públicos(7) y elementos sobre la producción de trabajos científicos que generen evidencia(8) e información suficiente para generar políticas públicas de calidad financiables por CONICYT en Chile(9), entre otros textos de interés.

El año 2005, en Chile, se inició una de las reformas de salud más grandes de su historia, al aplicar el Régimen de Garantías Explícitas en Salud -o Plan AUGE-, garantizando el acceso y cobertura a tratamientos de calidad para un número de patologías, con el objetivo de avanzar en Cobertura Universal(10) y reducir las inequidades en salud existentes en el territorio nacional(11). Como parte de dicha reforma, en la Ley 19.937(12) se establece que los establecimientos de salud que deseen otorgar prestaciones asociadas a GES, deben estar acreditados para este fin(13). Lo anterior hace necesario avanzar con efectividad, hacia la gestión de procesos hospitalarios con un nivel de calidad establecido, para reducir costos y aumentar la eficiencia en la entrega de prestaciones(14).

Pese a la importancia que la red asistencial pública tiene en el contexto dado por la Reforma, la deuda que mantienen los hospitales públicos por la falta de reajuste de los aranceles de prestaciones(7) no permite administrar apropiadamente las listas de espera(15) y los centros de urgencia, lo que afecta la satisfacción de los pacientes, quienes la evalúan como uno de los peores servicios del sistema nacional de salud(16).

Existen en Chile 186 hospitales públicos dependientes del Servicio Nacional de Salud, de los cua- les, 62 son de alta complejidad $(34 \%$ del total) (17). El Instituto Nacional del Tórax, hospital de altísima complejidad, ha estado en el centro de la agenda periodística —entre 2012 y 2014 - por informaciones que dan cuenta de tensiones internas que implican a parte de su equipo médico, en contraposición con la dirección $(18,19)$. Identificar los factores que provocan esas tensiones y los aspectos críticos que impactan en la gestión es parte de este trabajo.

Para realizar este trabajo, se recurrió a modelos previos de enfoque estratégico tradicional(20-22), en el marco de la utilización de los métodos e instrumentos conforme a la "Teoría fundamentada" $(23,24)$, que privilegia una mirada Bottom up, más que una Top down.

\section{Objetivos y metodología}

Sobre la base de lo contextualizado la pregunta de Investigación plantea la necesidad de determinar cuáles son los aspectos críticos que tiene el Instituto Nacional de Torax (INT), en cuanto hospital público de alta complejidad, que amenacen su quehacer, consecuencia de lo cual es preciso identificar los principales aspectos críticos contextuales y determinar su impacto en la gestión, desde la perspectiva de sus actores internos.

Corolario de lo anterior es exponer breves reflexiones sobre la dimensión ética de los procesos socio-organizacionales considerados, y converger con otras reflexiones sobre las exigencias éticas que se plantean, en el campo de la provisión de atención de salud, a nivel individual, colectivo y societal.

En lo metodológico, el artículo es parte de una investigación mayor de tipo cuali-cuantitativa $(25,26)$ que consta de dos partes y se propone responder las preguntas de la investigación, conceptualizando por medio de la Teoría Fundamentada o Grounded Theory $(27,28)$ a partir de la recogida sistemática de datos cualitativos y cuantitativos para ser analizados, por medio de triangulaciones, para obtener una interpretación de alcance medio que contribuya a explicar una hipótesis debidamente contrastada y representativa de un constructo Teórico de mayor densidad, robustez y consistencia(24). 


\section{Selección de la muestra}

El Equipo Directivo del hospital en estudio, junto a los investigadores, reunido en sesión de trabajo el lunes 8 de julio de 2013, definió los informantes calificados y la muestra para las entrevistas en profundidad(29) y los focus group (30), en el contexto del análisis FODA(21) en curso. En dicha sesión se acordó realizar tres entrevistas, específicamente al director del hospital; a la subdirectora de gestión de las personas, y a la subdirectora médica, como actores clave y representativos del nivel directivo. La coordinación de las reuniones se realizó con cada informante personalmente. Asimismo, se definió hacer dos focus group, uno de ellos compuesto por profesionales -18 de julio-y otro compuesto por técnicos y administrativos -19 de julio (tabla 1). Ambos coordinados con la subdirectora de gestión de las personas.

Tabla No 1: Participantes e instrumentos de recolección de información

\begin{tabular}{|c|c|c|c|}
\hline Actividad & Participantes & Estamento & Fecha \\
\hline Entrevista 1 & 1 & Directivo & 9 de julio \\
\hline Entrevista 2 & 1 & Directivo & 11 de julio \\
\hline Entrevista 3 & 1 & Directivo & 12 de julio \\
\hline $\begin{array}{c}\text { Focus Group } \\
1\end{array}$ & 10 & Profesional & 18 de julio \\
\hline $\begin{array}{c}\text { Focus Group } \\
2\end{array}$ & 8 & $\begin{array}{c}\text { Técnico y } \\
\text { administra- } \\
\text { tivo }\end{array}$ & Todos de julio \\
\hline $\begin{array}{c}\text { Participantes } \\
\text { en el proceso }\end{array}$ & $\begin{array}{c}9 \text { a 19 de } \\
\text { julio de } \\
2013\end{array}$ \\
\hline
\end{tabular}

Elaboración propia.

\section{Análisis de los datos}

El análisis de datos consideró, primero la reducción de estos por medio de procesos de categorización que implica la separación de unidades de texto; la identificación y clasificación de unidades; y finalmente, una síntesis y agrupamiento de términos clave $(31,32)$.

El segundo dispositivo, fue el de la disposición y transformación de datos lo cual supone conseguir un conjunto ordenado de información, normalmente presentada en forma espacial, abarcable y operativa para resolver las cuestiones de la investigación, considerando los resguardos necesarios en tanto y en cuanto, la disposición, conlleva un cambio en el lenguaje utilizado por quienes se expresan, generando una cierta transformación interpretada de datos(33,34). Es por ello que, en el presente estudio, la transformación de datos se manifiesta en tablas y mapas al final del análisis de material, del ámbito estudiado, en los que se presentan la dimensión, las categorías correspondientes a dicha dimensión con sus hallazgos simplificados, la valoración de cada tópico en el análisis $\operatorname{FODA}(21)$ y las categorías y/o hallazgos simplificados que se relacionan con la categoría referida, con el fin de aportar a la comprensión de la complejidad del discurso de los participantes en las entrevistas y de los grupos focales, en el contexto del fenómeno analizado.

\section{Resultados}

\section{Entrevistas(35)}

Las respuestas sistematizadas de los Directivos (Figura $\mathrm{N}^{\circ} 1$ ), representan su percepción acerca de las Amenazas y Oportunidades que, desde el entorno de la organización, le afectan o bien condicionan su quehacer. Los relatos se refieren, dentro de la dimensión reglas del juego, en primer lugar, a los cambios epidemiológicos y los nuevos factores de riesgo de la sociedad, los que sumados a la mayor proyección de vida de las personas, muchas de las cuales con multi-patologías, presiona al sistema a contar con nuevas tecnologías y al desarrollo de nuevas subespecialidades. Lo que obliga al INT, dada su alta especialización, a la adaptación y a la reinvención permanentes.

En segundo lugar, el contexto político y el consiguiente cambio en las líneas programáticas de gestión, es percibida por los entrevistados como una fuente constante de contingencia. Los cambios de autoridades implican nuevos desafíos para la organización y esto viene generalmente de la mano de cambios programáticos que obligan a cambiar el rumbo de lo que se ha venido haciendo.

Finalmente, en cuanto al sistema legal, el hecho de ser un hospital autogestionado y estar sometido a un proceso de acreditación, que debe certificar la calidad de los procesos, en un contexto de 
Figura $\mathrm{n}^{\circ}$ : análisis contextual desde las entrevistas a los directivos

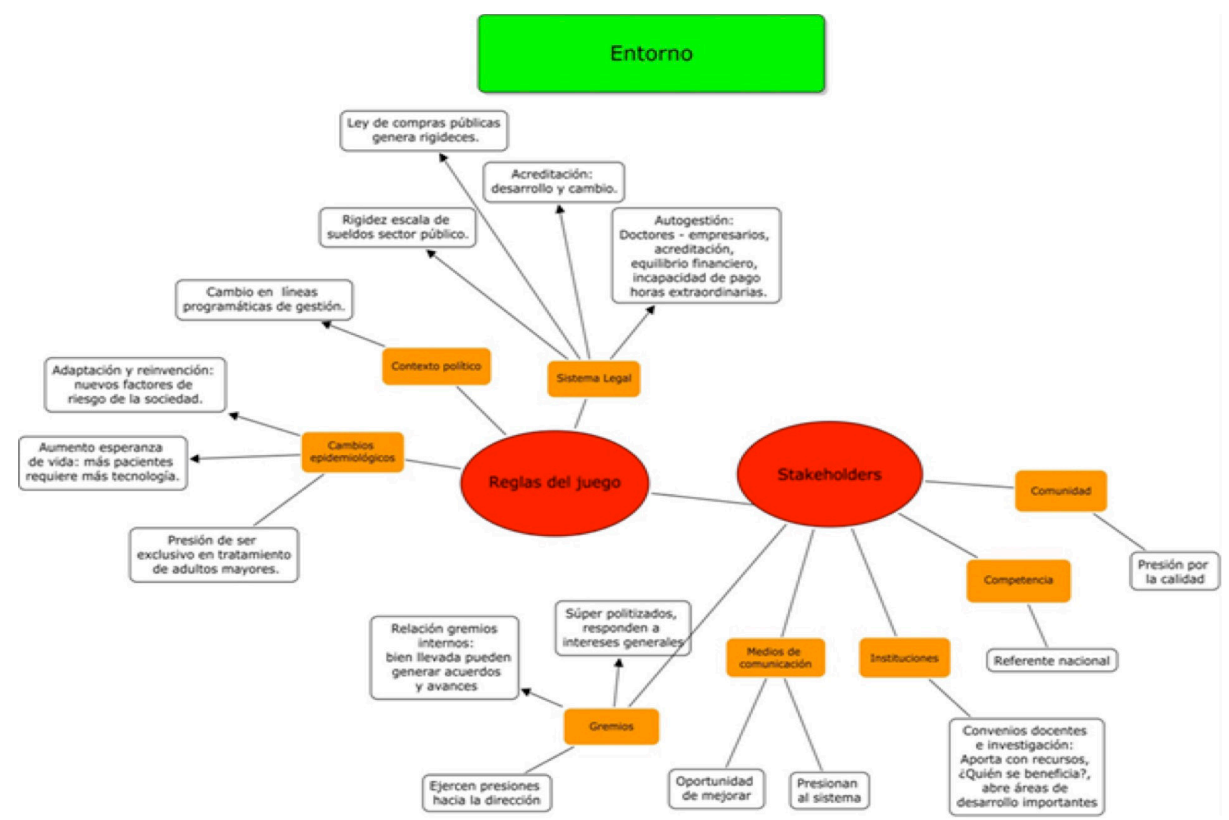

Elaboración propia, Fuente: Torres G., Informe Entrevistas en Profundidad Equipo Directivo INT, 2013.

rigideces, caracterizado por las regulaciones establecidas en la Ley de compras públicas y la definición de la escala de sueldos de sector público, que afecta la gestión del recurso humano, que por sus rigideces, termina generando Médicos - empresarios, que venden sus servicios fuera de su horario laboral, en calidad de empresa, afectando el desequilibrio financiero de la institución (figura 1).

En cuanto a la dimensión Stakeholders, a juicio de los entrevistados, un peso importante lo tiene la categoría competencia, puesto que el hecho de ser considerados "Referente Nacional", impone una fuerte presión de la competencia externa que los obliga a estar atentos para mantenerse en dicha condición. Esto implica cumplir metas institucionales, mantener la eficiencia y la calidad del servicio, con las limitaciones y rigideces propias del sistema.

Referente a las Instituciones relacionadas, los entrevistados señalan que los convenios de docencia e investigación con entidades académicas, representan una oportunidad como fuente adicional de ingresos, pero al ser coordinados y gestionados directamente por los médicos jefes de servicio, se desconoce cuál es el aporte real de recursos al hos- pital y quién se beneficia de ellos.

Respecto a la Comunidad y los medios de comunicación, los entrevistados señalan que existe una comunidad empoderada y bien informada que presiona por una buena calidad y mejor servicio, apoyada por el escrutinio de los medios de comunicación, que difunden y amplifican problemas circunstanciales que pueda haber en el INT. Lo anterior, imprime una presión adicional a las expectativas que genera el hecho de ser un referente nacional.

Finalmente, de acuerdo a la percepción de los entrevistados, una de las mayores fuentes de presión externa la constituyen los Gremios Nacionales representantes de los trabajadores y profesionales de la salud-, quienes ejercen influencia sobre los dirigentes gremiales internos que son "súper politizados" y responden a intereses generales independientemente de la realidad interna de la organización.

\section{Focus group (36)}

Las respuestas sistematizadas de los Profesionales, Técnicos y Administrativos (Figura $N^{\circ} 2$ ), repre- 
sentan su percepción acerca de las Amenazas y Oportunidades que, desde el entorno de la organización, les afectan o bien condicionan su quehacer.

Pese a que parece razonable a los participantes el empleo de las categorías FODA(21), como marco de la discusión para diagnosticar la organización, la estructura de sentido que organiza sus opiniones en torno a la valoración de lo que es relevante en la institución toca sólo tangencialmente el Ámbito Contextual, en el caso de los Profesionales, y no es abordado en absoluto por parte de los Técnicos y administrativos.

La primera mención en las narrativas de los participantes se refiere a la dimensión reglas del juego que impone el sistema legal —Categoría - a la organización. Dentro de ella lo relacionado con la Escala de sueldos del sector público y sus rigideces. Los profesionales hacen mención de este tema tomando la palabra no sólo por ellos sino representando a otros estamentos que son afectados, particularmente a funcionarios antiguos, auxiliares y paramédicos. Las diferencias entre los funcionarios antiguos, que trabajan en el marco del estatuto administrativo, versus los más nuevos, que trabajan a contrata u honorarios, con mayor grado y más sueldo pero sin beneficios, se percibe como un gatillante de malestar entre los trabajadores, produciendo deterioro en el clima organizacional.
En cuanto a la dimensión Cultura país, el grupo de profesionales percibe que hay aspectos de esta, caracterizada por la cultura del éxito, la Gestión por objetivos, los resultados y la producción a cualquier costo, que forman parte de la nueva manera de ver la gestión e incluso la vida en la sociedad actual y no es una cuestión exclusiva de la gestión del INT.

Por otra parte, la cultura piramidal del INT que establece jerarquías y estilos de dirección y liderazgo autoritarios, se daría en todos los hospitales. Este tipo de cultura está, de acuerdo a sus relatos, enraizada en la sociedad y particularmente en las organizaciones de salud de Chile, y sin la voluntad explícita de cambio desde la dirección, inevitablemente también les afectará a ellos.

La presentación de los resultados señalados en los párrafos anteriores, dan cuenta de cuáles son, a juicio de los entrevistados, los aspectos críticos que tiene el INT, a nivel de contexto. En la Tabla $\mathrm{N}^{\circ} 2$, se presenta en forma sintética las impresiones, de unos y otros, acerca de cuál es el impacto en la gestión que aquello genera, desde su propia perspectiva, asociando los distintos eventos a categorías relacionadas.

Figura $n^{\circ} 2$ : análisis contextual desde los focus group

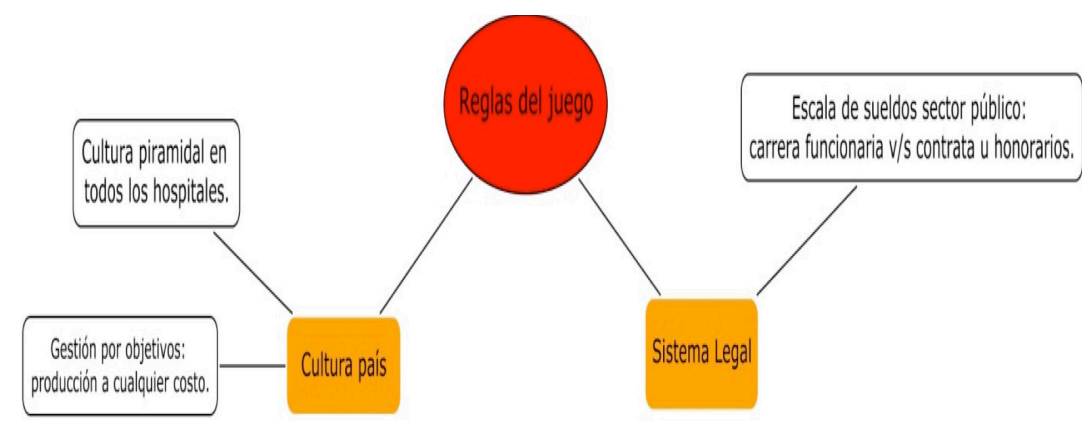

Elaboración propia. Fuente: (Torres G., Informe Focus Group INT, 2013). 
Aspectos críticos contextuales de un hospital de alta complejidad - Adrián Torres Canales et al.

\section{Tabla $\mathrm{N}^{\circ}$ 2: entrevista a actores relevantes del INT}

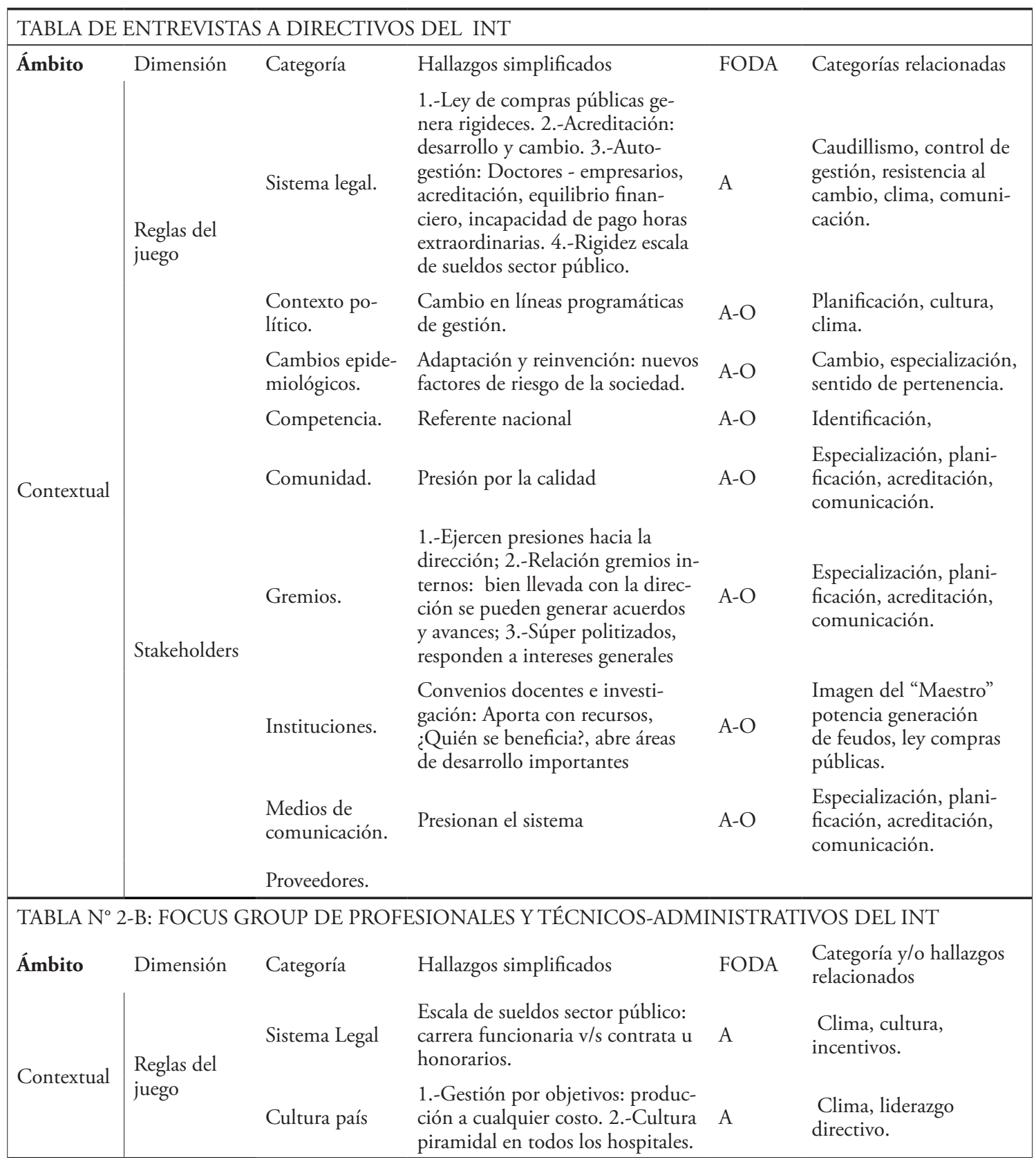

Elaboración propia, Fuentes: Informe de entrevistas(35) e informe de focus group(36). 


\section{Discusión}

El análisis se centró en la percepción de actores internos relevantes del Instituto Nacional del Tórax, acerca del impacto que tiene el entorno organizacional en su gestión. Las respuestas sistematizadas de los distintos grupos de entrevistados, demuestran que unos y otros perciben más amenazas que oportunidades de dicho entorno. Sin embargo, la percepción de los entrevistados no es la misma ni unívoca frente al fenómeno.

Los directivos hacen énfasis en que los cambios epidemiológicos y los nuevos factores de riesgo de la sociedad, presionan al sistema a la adaptación y a la reinvención permanentes. Pero que el contexto político, con el consiguiente cambio en las líneas programáticas de gestión, y un sistema legal y normativo, que impone fuertes obligaciones y está lleno de rigideces, termina generando anomalías como los Médicos - empresarios, que venden sus servicios fuera de su horario laboral, en calidad de empresa, afectando el desequilibrio financiero de la institución.

Lo anterior se ve agravado, primero, por el hecho de contar con una comunidad empoderada y bien informada que presiona por una buena calidad y mejor servicio, apoyada por el escrutinio de los medios de comunicación, y, en segundo lugar, por la presión externa de Gremios Nacionales representantes de los trabajadores y profesionales de la salud-, quienes ejercen influencia sobre los dirigentes gremiales internos que responden a intereses generales, independientemente de la realidad interna de la organización.

Los otros grupos de entrevistados, por su parte, especialmente los profesionales, estiman que el sistema legal, con la escala de sueldos propia del sector público, llena de rigideces para la gestión de recursos humanos, es un gatillante del malestar entre los trabajadores, produciendo deterioro en el clima organizacional.

No obstante, para estos lo más complejo son los efectos de la cultura país y la cultura organizacional del Instituto. En cuanto a la cultura país, se percibe que hay aspectos de esta, caracterizada por la cultura del éxito, la Gestión por objetivos, los resultados y la producción a cualquier costo, que forman parte de la nueva manera de ver la gestión pública e incluso la vida en la sociedad actual y no es una cuestión exclusiva de la gestión del INT.

Por otra parte, la cultura piramidal del INT que establece jerarquías y estilos de dirección y liderazgo autoritarios, es propia del sistema hospitalario. Este tipo de cultura está enraizada en la sociedad y particularmente en las organizaciones de salud de Chile, y sin la voluntad explícita de cambio desde la autoridad, inevitablemente les afectará también a ellos.

Se puede señalar, en consecuencia, que el predominio de una cultura país que potencia los derechos ciudadanos-comunidad empodera$\mathrm{da}(37,38)$, el derecho al acceso a la información y al escrutinio público de sus instituciones - medios de comunicación $(39,40)$ - y que instala un nuevo paradigma de administración del Estado —Nueva Gestión Pública(41)—, que concibe el uso eficiente de sus recursos; colisiona con la subcultura del INT, refractaria a los cambios, caracterizada por liderazgos históricos con agendas e intereses propios, la opacidad de sus procesos y gremios politizados.

La tensión entre la cultura país y la subcultura de este componente de la red asistencial pública, denota "cambios en la conciencia ética", que inciden tanto sobre los stakeholders como sobre las reglas del juego y representa un cambio en los patrones culturales, que incluye prácticas y discursos, y sobre todo la configuración de una matriz de exigencias más rigurosas sobre las decisiones que impactan en los demás actores.

De esta manera, se configuran dos focos relevantes de tensionamiento ético: la yuxtaposición de intereses públicos y privados, reflejada en el desarrollo de las prácticas médico-empresariales. Y por otro, más general, la dificultad de definir estándares y procedimientos normativos ante el choque de intereses específicos que caracteriza al sistema estudiado(42).

Una Reforma a la Salud viable requiere un mínimo común ético, que se exprese en sus contenidos explícitos, pero que más profundamente denote una conciencia compartida respecto del adecuado 
equilibrio entre derechos y deberes, y particularmente sobre las exigencias que esa Reforma impone a cada uno de sus componentes $(43,44)$.

Particularmente, en el nivel meso donde se ubica el INT, se puede plantear que un enfoque ético debiera hacer del establecimiento hospitalario un sujeto moral, cuya acción responda a claros criterios de responsabilidad social, y que potencie la excelencia profesional. Ello significa trabajar en la perspectiva delineada desde hace varios años por esfuerzos como los del Informe Dunning(45), o el Informe sobre Prioridades en Salud del Parlamento Sueco (1995) (46).

El estudio muestra que la organización hospitalaria en un contexto de reforma necesita resguardar los valores de quienes la integran, proveer un conjunto de servicios orientados a satisfacer su misión, y gestionar adecuadamente sus recursos. Enfrentados a la bipolaridad medios - fines, los problemas emergen cuando se desvanece la conciencia de los fines, y los medios pasan a suplantar esos fines. "La gestión no es sino un medio para lograr unos fines, y si hay diversas formas de medicina gestionada, habrá que optar por aquella que sea capaz de conjugar racionalidad económica y justicia” (47:71).

Los antecedentes presentados en el estudio, nos permiten hacer inferencias parciales de la evaluación diagnóstica realizada a partir de las percepciones de los entrevistados. Solo cuando tengamos a la vista los elementos organizacionales de la misma, podremos tener un resultado más concluyente acerca de todos los aspectos críticos que impactan la gestión del Instituto Nacional del Tórax.

En general, los resultados convergen con otros estudios, que han corroborado la ausencia de criterios éticos explícitos en los procesos de toma de decisiones sobre la asignación de recursos en el sector salud $(48,43)$. Debe considerarse el aporte del concepto de Responsabilidad Social en el campo de la salud, existiendo hoy día no solamente una cantidad de estudios sobre el tema $(49,50)$, sino también experiencias prácticas que deben ser conocidas y consideradas. Reviste un interés particular la coexistencia de una cultura jerárquica en las organizaciones, y particularmente en los hospitales, con una orientación a la obtención de resultados, asociados a valores de competencia y productividad. 


\section{Referencias}

1. Martín V. El fortalecimiento ético de las instituciones del ámbito público en América Latina. En Panamá; 2003. Recuperado de: http://unpan1.un.org/intradoc/groups/public/documents/CLAD/clad0047702.pdf

2. Naessens H. Ética pública y transparencia. Universidad de Santiago de Compostela, Centro Interdisciplinario de Estudios Americanistas Gumersindo Bustos; Consejo Espańol de Estudios Iberoamericanos; 2010: 2113-30. Recuperado de: https://halshs.archives-ouvertes.fr/halshs-00531532/document

3. Esteban EG. La teoría de los stakeholders: un puente para el desarrollo práctico de la ética empresarial y de la responsabilidad social corporativa. Veritas. Revista de Filosofía y Teología. 2007. Recuperado de: http://www.redalyc.org/ articulo.oa?id=291122924002

4. González Menéndez R. Humanismo y gestión de salud: pasado, presente y futuro. Rev Cuba Salud Pública 2006; 32(4).

5. Bustamante-Ubilla MA, Río-Rivero D, Carolina M, et al. Percepción de la motivación de los directivos intermedios en tres hospitales de la Región del Maule, Chile. Salud Pública Méx 2009; 51(5): 417-426.

6. PROTEGE (Chile). Insumos para un Plan de Fortalecimiento del Sector Salud. Perspectiva de los principales actores ministeriales. Santiago, Chile: Ministerio de Salud; 2009. Recuperado de: http://www.bcn.cl/catalogo/detalle_libro?bib=233124

7. Cid PC, Bastías SG. Evaluando la situación financiera de los hospitales públicos a partir de los costos actualizados de sus prestaciones. Rev Med Chile 2014; 142(2): 161-167.

8. Morales Asencio J, Gonzalo Jiménez E, Martín Santos F, et al. Evidence Based Public Health: resources on Efectiveness of Community Interventions. Rev Esp Salud Pública 2008; 82(1): 5-20.

9. Armas MR, Torres CA, Arriagada CJ, Muñoz PF, Salinas RR, Crocco AP. Proceso para priorizar las líneas de investigación esencial de interés para el Ministerio de Salud de Chile. Rev Méd Chile 2010; 138(4): 401-405.

10. Frenz P, Delgado I, Kaufman JS, Harper S. Achieving effective universal health coverage with equity: evidence from Chile. Health Policy Plan 2014; 29(6): 717-731.

11. Bastias G, Pantoja T, Leisewitz T, Zarate V. Health care reform in Chile. Can Med Assoc J 2008; 179(12): 1289-1292.

12. Chile. Ministerio de Salud. Modifica el D.L. No 2.763, de 1979, con la finalidad de establecer una nueva concepción de la autoridad sanitaria, distintas modalidades de gestión y fortalecer la participación ciudadana. LEY NUM. 19.937 2004. Recuperado de: http://www.leychile.cl/Navegar?idNorma=221629

13. Chile. Ministerio de Salud. Subsecretaría de Salud Pública. Reglamento orgánico de los establecimientos de salud de menor complejidad y de los establecimientos de autogestión en red. DTO. №38 2005. Recuperado de: http://web.minsal.cl/sites/ default/files/files/DTO-38_29-DIC-2005_Establecimientos\%20de\%20menor\%20complejidad\%20y\%20Autogestio$\operatorname{nados}(1) \cdot \mathrm{pdf}$

14. Rodríguez A. Towards a culture of quality hospitals. Medwave 2013; 13(09): e5810-e5810.

15. Letelier A, Cifuentes Rivas G. Waiting list in a public health facility in Santiago, Chile. Medwave 2014; 14(6): e6000.

16. Chile. Superintendencia de Salud. Departamento de Estudios y Desarrollo. Satisfacción y Calidad Percibida en la Atención de Salud Hospitalaria: Ranking de Prestadores Informe Global; 2013. Recuperado de: http://www.supersalud.gob.cl/ documentacion/569/articles-9005_recurso_1.pdf

17. Chile. Ministerio de Salud. Departamento de Estadísticas e Información de Salud. DEIS; 2015. Recuperado de: http:// www.deis.cl/

18. Villarrubia G. 31 profesionales del Hospital del Tórax revelan serias irregularidades que pagan los pacientes. CIPER Chile Centro de Investigación e Información Periodística 2012. Recuperado de: http://ciperchile.cl/2012/04/12/31profesionales-del-hospital-del-torax-revelan-serias-irregularidades-que-pagan-los-pacientes/

19. Villarrubia G. Estas denuncias están fundamentadas por problemas interpretativos de ciertos facultativos. CIPER Chile Centro de Investigación e Información Periodística 2015. Recuperado de: http://ciperchile. cl/2012/04/13/\%E2\%80\%9Cestas-denuncias-estan-fundamentadas-por-problemas-interpretativos-de-ciertosfacultativos $\% \mathrm{E} 2 \% 80 \% 9 \mathrm{D} /$

20. Münch L. Administración, gestión organizacional, enfoques y proceso administrativo. México: Pearson Educación; 2010.

21. David F. Conceptos de Administración Estratégica. México: Pearson Educación; 2003.

22. Majluf Sapag N. Los desafios de la gestión: de lo formal a lo sutil. Santiago, Chile: Aguilar Ediciones; 2011.

23. Charmaz K. Grounded Theory in the 21st Century: Applications for Advancing Social Justice Studies. En: The Sage handbook of qualitative research. Thousand Oaks California; United States: SAGE Publications; 2005: 507-535.

24. Gaete Quezada R. Reflexiones sobre las bases y procedimientos de la Teoría Fundamentada. Cienc Docencia Tecnol 2014; (48): 149-172.

25. Hernández R, Fernández-Collado C, Baptista P. Metodología de la investigación. México: McGraw Hill/Interamericana Editores; 2008. 
Aspectos críticos contextuales de un hospital de alta complejidad - Adrián Torres Canales et al.

26. Cook TD, Reichardt C. Métodos cualitativos y cuantitativos en investigación evaluativa. 1a ed. Madrid: Ediciones Morata; 1997.

27. Glaser BG, Strauss AL. The Discovery of Grounded Theory: Strategies for Qualitative Research. Aldine de Gruyter; 1967.

28. Requena AT, Planes VC, Miras RMS. Teoría fundamentada "Grounded theory": la construcción de la teoría a través del análisis interpretacional. España: CIS; 2006.

29. Gaínza V. La entrevista en profundidad individual. En: Canales M, (ed.). Metodologías de investigación social. Santiago, Chile: LOM Ediciones; 2006: 219-261.

30. Canales C. El Grupo de discusión y el Grupo Focal. En: Canales M, (ed.). Metodologías de Investigación Social. Santiago, Chile: LOM Ediciones; 2006: 265-287.

31. Abela J. Técnicas de Análisis de Contenido: Una revisión actualizada. Sevilla: Fundación Centro de Estudios Andaluces; 2002. Recuperado de: http://public.centrodeestudiosandaluces.es/pdfs/S200103.pdf

32. Revuelta F, Sánchez M. Programas de análisis cualitativo para la investigación en espacios virtuales de formación; 2015. Recuperado de: http://campus.usal.es/-teoriaeducacion/rev_numero_04/n4_art_revuelta_sanchez.htm

33. Ruiz Ruiz J. Análisis sociológico del discurso: métodos y lógicas. Forum: Qualitative Social Research 2009; 10(2). Recuperado a de: http://digital.csic.es/handle/10261/64955

34. Giraldo MG. Abordaje de la Investigación Cualitativa a través de la Teoría Fundamentada en los Datos. Ingeniería Industrial. Actualidad y Nuevas Tendencias 2011. Recuperado de: http://www.redalyc.org/articulo.oa?id=215021914006

35. Torres A. Informe Entrevistas en Profundidad Equipo Directivo INT. Santiago, Chile: ATC Gestión Integral de Organizaciones; 2013.

36. Torres A. Informe Focus Group INT. Santiago, Chile: ATC Gestión Integral de Organizaciones; 2013.

37. Escobar A. Participación ciudadana y políticas públicas. Una problematización acerca de la relación estado y sociedad civil en América Latina en la última década. Revista Austral de Ciencias Sociales 2004; 8: 97-108.

38. Arosteguy Pérez AI. Construcción de capital social comunitario y empoderamiento ciudadano. Última Década 2007. Recuperado de: http://www.redalyc.org/articulo.oa?id=19502607

39. Escudero MC. Comunicación para el empoderamiento y comunicación ecosocial. La necesaria creación de nuevos imaginarios. Perspect Comun 2009; 2(1): 146-158.

40. Montesinos Condo R. Medios de comunicación y ciudadanía. Revista de Investigación en Comunicación y Desarrollo 2011; 2(1): 16-24.

41. Morales Casetti M. Nueva Gestión Pública en Chile: Orígenes y efectos. Revista Ciencia Política 2014; 34(2): $417-438$.

42. Figueroa J. Médicos sin control: Los dueños de la salud pública en Chile. CIPER Chile Centro de Investigación e Información Periodística 2010. Recuperado de: http://ciperchile.cl/2010/09/27/medicos-sin-control-los-duenos-de-lasalud-publica-en-chile/

43. Martínez L. La equidad en la asignación de recursos en salud: una conciliación entre la ética de máximos y el mínimo decente. Clínica y Ciencia 2004; 1(1). Recuperado de: http://web.usach.cl/clinicayciencia/vol2_num1/jornada_intro.pdf

44. Mariñez F. Reflexiones sobre las éticas en la gestión pública. Administración \& Desarrollo 2011; 39(4): 85-98.

45. Cuervo J, Meneu R. Prioridades en atención sanitaria: informe para el Gobierno de Holanda (Informe Dunning). Barcelona: SG Editores; 1994.

46. Sweden Health Care and Medical Priorities Commission. Priorities in health care: ethics, economy, implementation: final report. Stockholm: The Commission; 1995.

47. Couceiro A. Los niveles de la justicia sanitaria y la distribución de los recursos. An Sis San Navar 2006; 29: 61-74.

48. Bedregal GP, Leal CV, Lavados MM, et al. Asignación de recursos en salud en Chile: Consideraciones éticas en la toma de decisión. Rev Méd Chile 2002; 130(2): 181-190.

49. Ortíz MI, Palavecino S. Bioética para una salud pública con responsabilidad social. Acta Bioethica 2005; 11(1): 65-76.

50. León F. Ética y responsabilidad social de las instituciones en el sistema de salud chileno. Temas de la Agenda Pública 2012; 54(7). Recuperado de: http://politicaspublicas.uc.cl/wp-content/uploads/2015/02/descargar-etica-y-responsabilidad-social-de-las-instituciones-en-el-sistema-de-salud-chileno.pdf

Recibido: 29 de septiembre de 2015

Aceptado: 19 de octubre de 2015 